\title{
The Smart Therapist: A Look to the Future
} of Smartphones and mHealth Technologies

\author{
in Psychotherapy
}


THE SMART THERAPIST AND mHEALTH TECHNOLOGIES

\begin{abstract}
mHealth refers to the use of mobile technologies in the provision of healthcare, and is an expanding field within psychotherapy research. Mobile technologies have the capacity to greatly enhance patient access, uptake, and engagement in psychological treatment. The purpose of the present paper was to define mHealth within clinical psychology, provide a review and discussion of the functionality of mHealth devices, current trends within the field of psychological mHealth, the challenges faced by researchers and clinicians in this field, and examine directions for future research. The main areas of research identified included the use of SMS technologies, ecological momentary assessment (EMA), online and App based interventions, and the recent integration of sensor and data mining technologies. Much of the research to date is lacking in methodological rigor, and reliance on standard research designs often employed in other areas of psychological research may constrain development of interventions. Research indicates that consumers want to engage in mHealth interventions, although only a limited number of empirically supported options currently exist. Future research incorporating innovative trial designs is required to meet consumer demand and provide users with evidence based treatment options.
\end{abstract}

Keywords: mobile phone, Smartphone, mHealth, e-mental health, technological adjuncts 
THE SMART THERAPIST AND mHEALTH TECHNOLOGIES

\section{The Smart Therapist: A Look to the Future of Smartphones and mHealth}

\section{Technologies in Psychotherapy}

Since the identification of mobile phones as a promising adjunctive technology to traditional psychotherapy (Boschen \& Casey, 2008; Clough \& Casey, 2011a) there has been a proliferation of research, with the field now commonly referred to as “mHealth”. We define mHealth as being any psychological or mental health intervention that is delivered or supported by the use of mobile technology, by means of a mobile phone or Smartphone. In addition, to be considered a mHealth intervention the purpose of the technology should be to enhance treatment or assessment, increase dissemination of interventions, or provide clinicians and clients with greater choice for accessing treatment materials or activities. We examine recent evidence concerning the emerging technologies associated with mobile phones and discuss the challenges associated with developing the evidence base to support mHealth. Specific focus is given to the applied use of these technologies and how they might benefit both patients and therapists.

\section{The Function of mHealth}

Mobile phones have the capacity to increase the availability and dissemination of evidence based treatment programs (Harrison et al., 2011; Proudfoot et al., 2013) and to enhance existing face to face therapy practices (Clough \& Casey, 2011a). Patient dropout or premature termination, non-attendance, and adherence to therapeutic tasks and behaviors remain problematic for even the most efficacious of face-to-face psychological treatment programs (e.g., Detweiler-Bedell \& Whisman, 2005; Melville, Casey, \& Kavanagh, 2007). 
THE SMART THERAPIST AND mHEALTH TECHNOLOGIES

Mobile phones may enhance patient engagement in therapy sessions, as well as adherence to therapeutic principles between therapy sessions (Casey \& Clough, in press; Clough \& Casey, 2011a, 2011b). In clinical practice, this may mean improved cost efficiency of services, better use of clinician time and resources, improved treatment outcomes and reduced risk of relapse. Furthermore, mHealth technologies may enable clients to require less frequent visits during a treatment program, benefitting those with limited access to services. This adjunctive use of mHealth technologies is also consistent with the recovery model of mental health (Department of Health, 2014). That is, the adjunctive use of these technologies is patient centred, gives clients greater treatment options, and facilitates ongoing intervention beyond a typical therapeutic program.

\section{Advantages of mHealth Technologies}

A primary advantage of the mobile phone is its ubiquity and penetration throughout society (Clough \& Casey, 2011a). At the end of 2013 an estimated $90 \%$ of adults living in the United States of America (USA) owned a mobile phone device, with individual ownership exceeding that of the standard desktop computer and tablet computer (Pew Research Centre, 2014). Importantly, mobile phone ownership is not restricted by socio-economic or demographic status (Harrison et al., 2011). Device ownership rates have been found to be equivalent between the general population and a community psychiatric sample (Carras, Mojtabai, Furr-Holden, Eaton, \& Cullen, 2014).

Other advantages and capabilities of the mobile phone include audio input and output capabilities, ability to send and receive text messages, voice calls, data input, output and storage, and typically low initial and maintenance cost (Clough \& Casey, 
THE SMART THERAPIST AND mHEALTH TECHNOLOGIES

2011a; Preziosa, Grassi, Gaggioli, \& Riva, 2009). The programmable mobile phone, or Smartphone, now forms the majority of personal mobile devices in many developed nations, including $58 \%$ of personal mobile phones in the USA (Pew Research Centre, 2014). Advantages of the Smartphone include internet access, camera, Bluetooth, voice and video calling, text and picture messaging, Global Positioning System (GPS), programmability, and the ability to download tailored software applications (Apps) to the device (Clough \& Casey, 2011a).

Recent advances in sensor technologies have created new opportunities for incorporating the Smartphone into clinical research and practice. Smartphones are built with a range of sensors, such as barometers, compass, accelerometers, proximity sensors, ambient light and noise sensors, temperature and humidity sensors, and eye tracking. Smartphones can be connected to external and wearable sensor devices, such as heart rate sensors, haptic and motor sensors, and even EEG headsets (e.g., Petersen, Stahlhut, Stopczynski, Larsen, \& Hansen, 2011; Shyamkumar, Oh, Banerjee, \& Varadan, 2013). Such sensors have led to a rise in what is now being termed the “quantified self” (Swan, 2012), with individuals able to measure and monitor a variety of health indicators and behaviors.

Smartphones are unique, in that the user often has a personal relationship with the device, with it forming integral links in the user's social, communication and often dating networks (Donner, 2008; Miller, 2012). It is this integration and importance of the Smartphone within the user's daily life that makes it such an attractive option for psychological researchers and practitioners. Many functions of the Smartphone may already be in use by clinicians, such as with the widely available applications such as "PE Coach” and "MyCompass” which can assist clinicians in working with clients on 
THE SMART THERAPIST AND mHEALTH TECHNOLOGIES

psychoeducation, monitoring, and coping skills. However further research is required to build the evidence base supporting these interventions.

\section{Current Trends in mHealth Research}

SMS technologies. Short Message Service (SMS) or text messaging is one of the more basic functions of mobile phones enabling SMS based interventions to be widely disseminated. These interventions can provide clients with brief reminders about coping strategies or appointments, as well as emergency contacts, encouragement and motivational messages. Many software programs are available which allow scheduling and bulk sending of SMS to individuals (e.g., www.esendex.com.au), providing a cost and time effective platform for intervention.

To date, SMS technology has been primarily researched in the periods before and after therapy. Several studies have demonstrated that text messages may be effective in increasing patient readiness to change and facilitating help seeking behaviors among participants not already engaged in treatment programs (Joyce \& Weibelzahl, 2011; Mason, Benotsch, Way, Kim, \& Snipes, 2014). There have however been mixed findings with regards to use of SMS as appointment reminders in psychology clinics. Whilst some studies have found positive effects associated with these interventions (Branson, Clemmey, \& Mukherjee, 2013; Sims et al., 2012), Clough and Casey (2014) found no beneficial effects associated with the messages and that patient dropout was in fact higher in the SMS condition. It was speculated that the SMS reminders may have inadvertently encouraged patients to take a more passive approach to appointment scheduling and recording (Clough \& Casey, 2014). The study provides important learning regarding the need for messages to be targeted, carefully integrated into practice, and empirically investigated for efficacy. 
THE SMART THERAPIST AND mHEALTH TECHNOLOGIES

Text messaging has been used to target patient engagement and adherence following discharge from inpatient programs. In an aftercare program for patients suffering bulimia nervosa, Bauer and colleagues (2012) found that remission rates were significantly greater for participants engaging in a weekly SMS intervention with tailored feedback, when compared to the treatment as usual condition. These findings indicate that SMS messages may be effective in facilitating the continuation of treatment gains once the treatment program has finished.

SMS has been used to encourage patient engagement during treatment programs. Aguilera and Munoz (2011) tested the feasibility of a daily text message adjunct to cognitive behavioral therapy (CBT) for depression. The 12 patients, who were participating in group therapy, received daily messages relating to mood monitoring, thought tracking, and pleasant event scheduling. The researchers found high patient acceptability and engagement with the SMS intervention, although efficacy was not examined. The SMS intervention was also tested on a small sample size, with no standardised measures of treatment satisfaction incorporated. However, in support of these findings Laursen (2010) found that regular text messages were generally well received and that the messages prompted reflection and awareness among young people engaging in programs for cannabis use. However, a quantitative evaluation of the intervention was not conducted. A recent study by Clough and Casey (submitted-b) found high patient satisfaction and acceptability for a daily SMS intervention as an adjunct to group therapy for anxiety disorders. The efficacy of the SMS intervention in this study (Clough \& Casey, submitted-b) is being further examined in an ongoing Randomised Controlled Trial (RCT).

Mobile phones for EMA. Mobile phones have been used for assessment and monitoring, primarily utilising EMA techniques. EMA is a non-invasive method for 
THE SMART THERAPIST AND mHEALTH TECHNOLOGIES

collecting real-time data from participants concerning symptomology, behavior, and thoughts, all whilst they interact in their daily lives (Axelson et al., 2003).

Among adolescents with high functioning Austism Spectrum Disorder moderate participant adherence was found for an EMA strategy delivered via mobile phone (Khor, Gray, Reid, \& Melvin, 2014). The EMA was delivered via an application and designed to assess stressors and coping over a two week period. The study found that on average the adolescents responded to approximately $61 \%$ of prompts to complete the assessments, with moderate concurrent validity also established. Axelson et al. (2003) and Matthews et al. (2008) also examined the use of mobile phones for conducting EMA among adolescent populations. Both studies found high acceptability and feasibility for the interventions.

These results are supported by Tilley and Rees (2014) who used mobile phones to prompt patients with Obsessive Compulsive Disorder (OCD) to engage in EMA activities. The EMA intervention produced a lower number of obsessions and compulsions than reported on the Yale-Brown Obsessive-Compulsive Scale (YBOCS), but produced additional types that were not identified on the Y-BOCS. However this study reports the use of this intervention within a small sample size of three clinical case studies. Further research is required to demonstrate generalisability.

Overall, the studies to date indicate that the mobile phone is a particularly promising device to assist in the monitoring of patient symptoms and behaviors through EMA. The device is generally well accepted by participants and may improve patient monitoring when compared to pen and paper strategies. However, more research involving larger sample sizes and structured EMA interventions is needed to comprehensively assess these types of monitoring interventions. Furthermore, given that many of these studies utilised only brief periods for EMA, it is unclear whether 
THE SMART THERAPIST AND mHEALTH TECHNOLOGIES

greater adherence to mobile phone EMA is the result of the novelty of using the mobile phone for this purpose. Further research is needed to assess whether patient engagement and acceptability remain high when EMA interventions extend over longer periods of time.

Apps and online interventions. The mobile phone is increasingly used for the delivery of online interventions. These types of interventions allow participants to access a broad range of material at the touch of button, which can be updated by the therapist or programmer and completed by the participant whenever they choose. Many individuals also indicate a preference for obtaining health related information through the internet (Casey, Joy, \& Clough, 2013; Klein \& Cook, 2010). These types of interventions are designed for Smartphones rather than standard mobile phones, and may be accessed through Internet browsers on the devices or through downloadable Apps.

An online Smartphone intervention was used in the aftercare of women hospitalised for chronic pain (Kristjánsdóttir et al., 2013). The 140 women were randomised to Smartphone or treatment as usual conditions for a two-week aftercare period. The Smartphone intervention consisted of diaries and reflection activities uploaded to a website and made available for therapist feedback. SMS reminders, audio files and guided mindfulness were also utilised. On completion of the intervention, participants in the Smartphone group reported significantly less catastrophizing and significantly greater acceptance of pain. In contrast, the control group deteriorated over the same period, indicating that the Smartphone may be an effective adjunct during aftercare. However, the design of this intervention was unable to identify the active components of treatment. 
THE SMART THERAPIST AND mHEALTH TECHNOLOGIES

A combined Smartphone intervention was also used for a behavioral parent training program (Jones et al., 2013). Participants viewed a series of videos on parenting skills (viewed on the Smartphone), completed brief daily surveys, received text message reminders, submitted video recorded home practice of skills, and a received a midweek video call from a practitioner. The enhanced treatment program improved child outcomes and families required fewer sessions to complete the parenting program (Jones et al., 2013). These results indicate that the adjunctive use of Smartphone interventions may also be cost effective for services.

Smartphone interventions have been used to complement e-mental health interventions delivered by standard computer. By means of an RCT, Proudfoot and colleagues (2013) investigated the efficacy of “myCompass”, a mobile and computer based intervention for mild to moderate depression, anxiety and stress. Participants completed online CBT modules accessible by computer, and were able to engage in EMA, receive feedback, reminders, and helpful facts and motivational statements to their Smartphone device. Compared to attention control and waitlist conditions, participants in the intervention displayed greater reductions in depression, stress and anxiety with small to moderate effect sizes observed between groups. Although effect sizes were smaller and dropout rate (48\%) higher than most published trials of traditional CBT interventions, the reach of the intervention needs to be considered alongside the efficacy. Reach refers to the proportion of a target population who access a service (Halford \& Casey, 2010). Many psychological interventions are compromised by low reach. As a publicly available program, “myCompass” has demonstrated high reach. As such even small effect sizes when implemented on a large scale can significantly improve public health. 
THE SMART THERAPIST AND mHEALTH TECHNOLOGIES

Combining internet and Smartphone capabilities in the delivery of online interventions, such as with the approach used in "myCompass” (Proudfoot et al., 2013), may be able to increase patient engagement with programs by increasing the options available for access. Watts et al. (2013) compared the efficacy of an online treatment program for depression when delivered by either fixed (desktop) computer or by mobile phone/ tablet computer. The study found equivalence between the modalities of delivery, with both groups showing significant improvements in treatment over the course of the program and with gains maintained at follow up assessment. These results provide preliminary support for the use of online programs on mobile phone and tablet devices and as the researchers note provide patients with greater choice regarding their preferred delivery device, and increase convenience, accessibility and privacy.

Online interventions for Smartphones are increasingly being programmed as Apps. It was estimated that in 2013 there were over 10,000 mental health Apps available for download (Ben-Zeev et al., 2013). In a recent systematic review of research examining mental health Apps for mobile devices Donker et al. (2013) found only eight papers, describing five Apps, met their inclusion criteria (such as having a control group or pre to post-test design) for review. Four of the Apps were for use with support from a mental health professional. The intention-to-treat effect sizes for these Apps ranged from weak to strong, with the authors concluding that whilst mHealth Apps within mental health show promise, there is lack of scientific evidence concerning their efficacy.

Ben-Zeev et al. (2013) report on the development and pilot testing of a Smartphone App (FOCUS) designed to assist in self-management for patients with schizophrenia. Participants engaged in regular self-assessments by means of multiple- 
THE SMART THERAPIST AND mHEALTH TECHNOLOGIES

choice questions, with the App then providing the participant with illness management tips related to areas such as medication adherence, mood, and sleep.

Daily use of the App was reported by 58\% of users and acceptance was high. A later study of FOCUS examined usage over a one month period among 33 individuals with schizophrenia (Ben-Zeev, Brenner, et al., 2014). High usage of the App was found among completers, as well as high ratings of participant acceptability and usability. Significant reductions in psychotic symptoms, depression, and general psychopathology were observed following the one-month period. The study did not however contain a control or comparison condition.

Smartphone Apps also exist for the treatment of depression (e.g., Harrison et al., 2011), anxiety (e.g., Clough \& Casey, submitted-a; Reger et al., 2013), addictions (e.g., Gustafson et al., 2014) and many other mental health and related conditions (Casey et al., 2014). Similar findings of high acceptability and feasibility have been found in other studies of these Apps (e.g., Clough \& Casey, submitted-a). However, there is a lack of rigorous, randomised testing of these programs.

Sensor Technologies. Recently, research has begun to examine the uses of Smartphone sensor technology in psychotherapy. This is an emerging field, but one that will likely expand and make the transition to clinical practice in coming years.

Burns and colleagues (2011) describe the development and pilot testing of a sensor informed Smartphone intervention for depression, “Mobilyze!”. The intervention utilised machine learning (data mining techniques) whereby information from the participant's Smartphone sensors (such as GPS, ambient light and recent calls) were obtained and paired with EMA responses. During the machine learning period participants were prompted to answer questions relating to activities and mood states via the Smartphone, with responses paired with sensor data. A model was 
THE SMART THERAPIST AND mHEALTH TECHNOLOGIES

created whereby the sensor data was used to make predictions about the participant's mood, motivation, cognitive states, and social and environmental contexts. The machine learning models provided accurate location and context predictions, but predictions regarding mood were poor. Poor accuracy relating to prediction of mood states was thought to be due to an insufficient amount of paired user-sensor data. The Smartphone sensing and EMA intervention was delivered concurrently with an interactive website for behavioral skills training and email and telephone support for each participant. Over the eight-week trial period participants displayed clinically significant improvement and high satisfaction with the intervention. Although the machine learning component of the intervention was only moderately successful, continued development of the program may assist patients in understanding links between behaviors and mood as well as how they spend their time.

Smartphone sensor technology has also been used in the aftercare of patients treated for alcoholism (Gustafson et al., 2014). A Smartphone App was developed which included audio guided relaxation, a sobriety counter, daily motivational text messages, a panic button to receive additional support if the patient felt they were at risk of relapsing, and alerts to advise the patient of healthy and drug free events taking place in their city. The App also made use of the Smartphone’s GPS system. Patients listed areas and addresses of high risk for personal relapse. The GPS sensor then tracked when patients were approaching areas of high risk and initiated an alert to provide patients with support. The individual component effect of the sensor system was not investigated. However, in comparison to a treatment as usual group patients with access to the Smartphone intervention demonstrated an increased likelihood of abstinence and significantly fewer "risky" drinking days throughout the eight-month 
THE SMART THERAPIST AND mHEALTH TECHNOLOGIES

intervention and four month follow up period. This study illustrates the possibilities for Smartphones to increase support for clients following a period of psychotherapy.

In non-clinical samples sensors have also been used to measure conversational techniques and characteristics (e.g., Rahman et al., 2011), for monitoring activity in weight loss interventions (e.g., Shuger et al., 2011), and other health based interventions. Whilst research in this area is currently limited, sensor based technologies are likely to provide clinicians and researchers with opportunities for creative and ecologically valid learning and therapeutic intervention techniques.

\section{Limitations and Difficulties with mHealth Research}

Despite promising preliminary findings in this field, research is limited and that which does exist is often underpowered or lacking methodological rigor. Researchers within mHealth are presented with many challenges unique to this field, which often hamper the progress of technologically based research projects.

One particular challenge lies in the implementation of the RCT, which is considered to be the gold standard of research designs. The RCT is championed for its use in assessing treatment efficacy and inferring causality. Ioannidis (1998) identified the medium time from grant application to publication for RCTs was 7 years, with 5.5 years between initial enrolment and publication. During this 7 year median time frame, it is likely that technology may become superseded or obsolete (Kumar et al., 2013). The progression from research to clinical practice is even slower. It has been estimated that from project concept to community implementation of techniques takes on average 17 years (Riley, Glasgow, Etheredge, \& Abernethy, 2013).

Riley et al. (2013) argue for a number of changes to standard research designs. These changes are aimed at research being conducted with relevance to the coined 
THE SMART THERAPIST AND mHEALTH TECHNOLOGIES

"R3"; that research needs to be more rapid, responsive and relevant. These recommendations include replacing traditional pilot testing with iterative $\mathrm{N}=1$ designs, collaboration with community and industry stakeholders to enable speedier recruitment of participants and subsequent adoption of practices, and optimisation strategies such as sequential multiple assignment. Given that many of the suggested research designs can still infer causality and demonstrate efficacy (such as if employing iterative $\mathrm{N}=1$ cases with a multiple baseline design for pilot testing), these recommendations provide an alternative to the traditional RCT methodology.

Another challenge in mHealth research concerns the technical difficulties often associated in using new technologies. Programming for the Smartphone can be complex, with a range of platforms currently in use. Many researchers and clinicians within psychology may have limited exposure or knowledge of programming or software design. As a result inadequate time and scientific effort is often devoted to the design and development of mHealth interventions (Ben-Zeev et al., 2013). BenZeev, Schueller, et al. (2014) argue that for mHealth research to be successful collaboration with technologists is required at all phases of the project.

Greater effort is needed in providing clinicians with opportunities for training and access to developed mobile technologies. Therapist comfort with technology will undoubtedly influence patient willingness to engage with these interventions. Training for therapists on the uses of technologies and ways to incorporate mHealth into their practice may facilitate uptake and engagement. Such training would also be helpful for those therapists wanting to incorporate mobile technologies into their practice who may need guidance in starting in this field. As with other developments in this field, training interventions need to be carefully designed and empirically validated. Initially this will likely involve greater examination of therapists’ perceived 
THE SMART THERAPIST AND mHEALTH TECHNOLOGIES

barriers to mHealth use, with this information then used to inform the development of training programs.

The gap between research and practice in this field is substantial. The recommendations outlined above may help to bridge this gap. The incorporation of structured, multiple baseline case research may enable more research to be conducted in applied settings. This incorporation would likely lead to the development of adjunctive interventions which are guided by clinical need, as well as greater and faster uptake of technologies into clinical practice. What is known from current research is that the marketplace and consumers are not waiting for scientific validation before adopting mHealth technologies (Kumar et al., 2013). It is therefore imperative that strategies are implemented so that consumers may be provided with empirically supported mHealth interventions.

\section{Future Directions}

Mobile technologies are developing at increasingly rapid rates. Moore’s Law argues that electronic storage capacities will double every two years, whilst Kryder's Law argues that the cost per unit for electronic storage will halve every two years (Miller, 2012). These factors, combined with the further development of internal and external mobile sensor technologies, indicate that the Smartphone will continue to be an increasingly powerful and affordable handheld computational device.

Mobile sensor technologies are perfectly suited for incorporation into biofeedback interventions, particularly "wearables” or body worn sensors. For example, wearable sensor devices may be able to monitor a patient's heart rate and breathing, with this information then wirelessly transmitted back to a patient's 
THE SMART THERAPIST AND mHEALTH TECHNOLOGIES

Smartphone device. An accompanying App would then present patients with their relevant data, and provide feedback and guidance on skills.

Apps used as adjuncts to provide therapists with real time summaries of patient data, may lead to improved outcomes and usage of these programs.

Ecologically valid sensor data may help therapists to better understand patient difficulties, personalise treatment plans, and monitor patient engagement and progress. For example, sensor data may help a patient and therapist understand the sleeping patterns of a patient suffering insomnia, or the changes in physical arousal that occur during prolonged exposure tasks. In the latter example, wearable sensors could track a patient’s physiological responses during an exposure task. This may helping the therapist and client better understand the patient's response to a stimulus, and provide feedback regarding anxiety reduction and habituation during the task. Patients would then use the same procedure to guide independent exposures between therapy sessions, with therapists able to review this data remotely or with the patient in the next session. Such self monitoring may increase patients emotional awareness and provide a means for patients to track their symptoms and review their progress.

Context aware programs involving machine learning offer a multitude of possibilities for creating interventions that target patient difficulties as they occur in their everyday lives.. These interventions may assist patients in understanding patterns and relationships between their thoughts, feelings, behaviors and activities. In addition patients may be able to be provided with real time support when in high risk situations or when the individual's sensor patterns indicate that they may be experiencing mood difficulties or engaging in problematic behaviors. When considering parent training interventions, through a period of machine learning the program may identify high risk times, places, or even identify changes in ambient 
THE SMART THERAPIST AND mHEALTH TECHNOLOGIES

noise within the family context. Once the program is able to make adequate context predictions based on the sensor data, an intervention may be delivered during high risk or stress periods. The patient could receive messages offering support, tips, videos, or reminders about strategies discussed in session.

Smartphone devices may also be able to replace current virtual reality devices, which can be expensive and cumbersome. Devices are available which allow a basic virtual reality program to be run from the user's Smartphone device (e.g. Samsung's “Gear VR”). These systems will continue to improve with the Smartphone likely to be able handle complex virtual reality programs in the near future. In addition, the Smartphone may connect to external devices to provide haptic, kinematic, or olfactory simulations to create an immersive virtual reality experience. Virtual reality therapies have demonstrated efficacy across a range of disorders (Turner \& Casey, in press). The Smartphone may enable this style of therapy to be more widely disseminated and used by the practicing clinician, as well as for patient practice to occur more regularly between sessions.

The Smartphone may be useful in increasing patient access to online therapies, through mobile delivery that allows users to access programs at times when they would not previously have been able to do so. For example, a patient may engage in a homework activity on their Smartphone whilst commuting or in public places. Other suggested uses for the Smartphone include using the sensors and microphone to provide patients with feedback on social skills (e.g. turn taking and speech qualities) in conversations, or using wearable sensors to provide feedback on posture in pain management programs (Morris \& Aguilera, 2012).

The emerging field of research in mHealth also gives rise to new ethical considerations for researchers and clinicians. Context aware systems that rely on data 
THE SMART THERAPIST AND mHEALTH TECHNOLOGIES

mining and machine learning from the device sensors involve the collection of large quantities of personal information. Participants need an understanding of the types of information collected by the system (location, ambient noise, recent calls) and appropriate consent mechanisms must be developed. Particular care should be given to the de-identification of sensor data, secure transmission and storage of this information, feedback to patients, and risk management protocols. Clinicians need to consider patient privacy, and tailor the use of technologies to meet individual patient comfort and expectations. An intervention that is perceived as acceptable by one patient, may be perceived as burdensome or intrusive by another. Clinicians will need to remain flexible and approach the technologies from a patient centred model. Ongoing discussion concerning best practice standards for the ethical use and implementation of these technological interventions is required.

\section{Conclusions}

This paper has provided a discussion of the function, technologies, and research within the field of psychological mHealth and examined the challenges associated with mHealth research. A review of the literature indicates that a small number of empirically supported interventions exist, with many other interventions demonstrating preliminary efficacy. Research within this field includes the use of SMS interventions, online and App based interventions, mobile technologies for EMA, and sensor based interventions. Considerable methodological limitations and difficulties exist within this field of research.

Limitations include a lack of fit between the traditional research designs (such as the RCT) and the evolving nature of technologies, as well as a need for collaboration between researchers, mental health professionals, users, and 
THE SMART THERAPIST AND mHEALTH TECHNOLOGIES

technologists. Currently there is a considerable gap between the consumer desire for these interventions and the empirically supported options available to them.

Consumers are not likely to wait for interventions to pass an RCT trial period before use, and other research designs may be necessary to meet this need. The development and incorporation of innovative study designs will likely accelerate research and adoption of mHealth technologies.

There are many exciting possibilities for the future of mHealth within psychotherapy. The Smartphone offers the opportunity for interventions to be delivered through one integrated system. One device can engage participants in virtual reality, EMA, deliver content, videos, reminders, make predictions regarding the user's state, and provide in the moment therapy and support. The Smartphone is a device that has substantial capacity to improve an individual's experience of psychotherapy. 
THE SMART THERAPIST AND mHEALTH TECHNOLOGIES

\section{References}

Aguilera, A., \& Munoz, R. F. (2011). Text Messaging as an Adjunct to CBT in LowIncome Populations: A Usability and Feasibility Pilot Study. [Article]. Professional Psychology-Research and Practice, 42(6), 472-478. doi: 10.1037/a0025499

Axelson, D. A., Bertocci, M. A., Lewin, D. S., Trubnick, L. S., Birmaher, B., Williamson, D. E., .. . Dahl, R. E. (2003). Measuring mood and complex behavior in natural environments: Use of ecological momentary assessment in pediatric affective disorders. Journal of Child and Adolescent Psychopharmacology, 13(3), 253-266.

Bauer, S., Okon, E., Meermann, R., \& Kordy, H. (2012). Technology-Enhanced Maintenance of Treatment Gains in Eating Disorders: Efficacy of an Intervention Delivered via Text Messaging. [Article]. Journal of Consulting and Clinical Psychology, 80(4), 700-706. doi: 10.1037/a0028030

Ben-Zeev, D., Brenner, C. J., Begale, M., Duffecy, J., Mohr, D. C., \& Mueser, K. T. (2014). Feasibility, Acceptability, and Preliminary Efficacy of a Smartphone Intervention for Schizophrenia. Schizophrenia Bulletin. doi:

10.1093/schbul/sbu033

Ben-Zeev, D., Kaiser, S. M., Brenner, C. J., Begale, M., Duffecy, J., \& Mohr, D. C. (2013). Development and usability testing of FOCUS: A smartphone system for self-management of schizophrenia. Psychiatric Rehabilitation Journal, 36(4), 289-296. doi: 10.1037/prj0000019

Ben-Zeev, D., Schueller, S. M., Begale, M., Duffecy, J., Kane, J. M., \& Mohr, D. C. (2014). Strategies for mHealth Research: Lessons from 3 Mobile Intervention 
THE SMART THERAPIST AND mHEALTH TECHNOLOGIES

Studies. Administration and Policy in Mental Health and Mental Health Services Research, 1-11.

Boschen, M. J., \& Casey, L. M. (2008). The Use of Mobile Telephones as Adjuncts to Cognitive Behavioral Psychotherapy. Professional Psychology-Research and Practice, 39(5), 546-552. doi: 10.1037/0735-7028.39.5.546

Branson, C. E., Clemmey, P., \& Mukherjee, P. (2013). Text message reminders to improve outpatient therapy attendance among adolescents: A pilot study. Psychol Serv, 10(3), 298-303. doi: 10.1037/a0026693

Burns, M. N., Begale, M., Duffecy, J., Gergle, D., Karr, C. J., Giangrande, E., \& Mohr, D. C. (2011). Harnessing context sensing to develop a mobile intervention for depression. Journal of Medical Internet Research, 13(3).

Carras, M. C., Mojtabai, R., Furr-Holden, C. D., Eaton, W., \& Cullen, B. A. M. (2014). Use of Mobile Phones, Computers and Internet Among Clients of an Inner-City Community Psychiatric Clinic. Journal of Psychiatric Practice ${ }^{\circledR}$, 20(2), 94-103 110.1097/1001.pra.0000445244.0000408307.0000445284.

Casey, L. M., \& Clough, B. A. (in press). Making and keeping the connection: Improving consumer attitudes and engagement in e-mental health interventions. In G. Riva, W. Wiederhold \& P. Cipresso (Eds.), The Psychology of Social Networking: Communication, Presence, Identity and Relationships in Online Communities. Open Access: Versita.

Casey, L. M., Clough, B. A., Mihuta, M. E., Green, H., Usher, W., James, D. A., . . . Laakso, E. (2014). Computer-based interactive health communications for people with chronic disease. Smart Homecare Technology \& TeleHealth, 2. 
THE SMART THERAPIST AND mHEALTH TECHNOLOGIES

Casey, L. M., Joy, A., \& Clough, B. A. (2013). The Impact of Information on Attitudes Toward E-Mental Health Services. Cyberpsychology, Behavior, and Social Networking, 16(8), 593-598. doi: 10.1089/cyber.2012.0515

Clough, B. A., \& Casey, L. M. (2011a). Technological Adjuncts to Enhance Current Psychotherapy Practices: A Review. Clinical Psychology Review, 31(3), 279292. doi: 10.1016/j.cpr.2011.03.006

Clough, B. A., \& Casey, L. M. (2011b). Technological Adjuncts to Increase Adherence to Therapy: A Review. Clinical Psychology Review, 31, 697-710. doi: 10.1016/j.cpr.2011.03.006

Clough, B. A., \& Casey, L. M. (2014). Using SMS Reminders in Psychology Clinics: A Cautionary Tale. Behav Cogn Psychother, 4, 1-12. doi: $10.1017 / \mathrm{S} 1352465813001173$

Clough, B. A., \& Casey, L. M. (submitted-a). Therapy on the move: The development of a therapeutic Smartphone application. Griffith University. Brisbane, Australia.

Clough, B. A., \& Casey, L. M. (submitted-b). Using Daily SMS to Support Adherence to Homework in Psychotherapy: Client Acceptability and Satisfaction with Treatment. Griffith Univeristy. Brisbane, Australia.

Department of Health. (2014). National framework for recovery-oriented mentalh health services Retrieved October 13, 2014, from http://www.health.gov.au/internet/main/publishing.nsf/Content/mental-pubs$\underline{\text { n-recovfra }}$

Detweiler-Bedell, J. B., \& Whisman, M. A. (2005). A lesson in assigning homework: Therapist, client, and task characteristics in cognitive therapy for depression. 
THE SMART THERAPIST AND mHEALTH TECHNOLOGIES

Professional Psychology-Research and Practice, 36(2), 219-223. doi: 10.1037/0735-7028.36.2.219

Donker, T., Petrie, K., Proudfoot, J., Clarke, J., Birch, M.-R., \& Christensen, H. (2013). Smartphones for smarter delivery of mental health programs: a systematic review. Journal of Medical Internet Research, 15(11). doi: 10.2196/jmir.2791

Donner, J. (2008). Research approaches to mobile use in the developing world: A review of the literature. The information society, 24(3), 140-159.

Gustafson, D. H., McTavish, F. M., Chih, M.-Y., Atwood, A. K., Johnson, R. A., Boyle, M. G., .. . Dillenburg, L. (2014). A smartphone application to support recovery from alcoholism: a randomized clinical trial. JAMA psychiatry.

Halford, W. K., \& Casey, L. M. (2010). Taking it to the people: Using technology to enhance the impact of couple relationship education. In K. Hahlweg, M.

Grawe \& D. Baucom (Eds.), Enhancing couples: The shape of couple therapy to come (pp. 111-127). Gottingen, Germany: Hogrefe.

Harrison, V., Proudfoot, J., Wee, P. P., Parker, G., Pavlovic, D. H., \& Manicavasagar, V. (2011). Mobile mental health: Review of the emerging field and proof of concept study. Journal of Mental Health, 20(6), 509-524. doi: doi:10.3109/09638237.2011.608746

Ioannidis, J. A. (1998). EFfect of the statistical significance of results on the time to completion and publication of randomized efficacy trials. JAMA, 279(4), 281286. doi: 10.1001/jama.279.4.281

Jones, D. J., Forehand, R., Cuellar, J., Kincaid, C., Parent, J., Fenton, N., \& Goodrum, N. (2013). Harnessing innovative technologies to advance children's mental 
THE SMART THERAPIST AND mHEALTH TECHNOLOGIES

health: Behavioral parent training as an example. Clinical psychology review, 33(2), 241-252.

Khor, A. S., Gray, K. M., Reid, S. C., \& Melvin, G. A. (2014). Feasibility and validity of ecological momentary assessment in adolescents with high-functioning autism and Asperger's disorder. Journal of Adolescence, 37(1), 37-46. doi: http://dx.doi.org/10.1016/j.adolescence.2013.10.005

Klein, B., \& Cook, S. (2010). Preferences for e-mental health services amongst an online Australian sample. Electronic Journal of Applied Psychology, 6, 28-39.

Kristjánsdóttir, Ó. B., Fors, E. A., Eide, E., Finset, A., Stensrud, T. L., van Dulmen, S., ... Eide, H. (2013). A smartphone-based intervention with diaries and therapist-feedback to reduce catastrophizing and increase functioning in women with chronic widespread pain: randomized controlled trial. Journal of Medical Internet Research, 15(1), e5.

Kumar, S., Nilsen, W. J., Abernethy, A., Atienza, A., Patrick, K., Pavel, M., . . . Spruijt-Metz, D. (2013). Mobile health technology evaluation: the mHealth evidence workshop. American journal of preventive medicine, 45(2), 228-236.

Laursen, D. (2010). Counseling Young Cannabis Users by Text Message. [Article]. Journal of Computer-Mediated Communication, 15(4), 646-665. doi: 10.1111/j.1083-6101.2009.01510.x

Matthews, M., Doherty, G., Sharry, J., \& Fitzpatrick, C. (2008). Mobile phone mood charting for adolescents. British Journal of Guidance \& Counselling, 36(2), 113-129. doi: 10.1080/03069880801926400

Melville, K. M., Casey, L. M., \& Kavanagh, D. J. (2007). Psychological treatment dropout among pathological gamblers. Clinical Psychology Review, 27, 944958. doi: 10.1016/j.cpr.2007.02.004 
THE SMART THERAPIST AND mHEALTH TECHNOLOGIES

Miller, G. (2012). The Smartphone Psychology Manifesto. Perspectives on Psychological Science, 7(3), 221-237. doi: 10.1177/1745691612441215

Morris, M. E., \& Aguilera, A. (2012). Mobile, social, and wearable computing and the evolution of psychological practice. Professional Psychology: Research and Practice, 43(6), 622.

Petersen, M., Stahlhut, C., Stopczynski, A., Larsen, J., \& Hansen, L. (2011). Smartphones Get Emotional: Mind Reading Images and Reconstructing the Neural Sources. In S. D’Mello, A. Graesser, B. Schuller \& J.-C. Martin (Eds.), Affective Computing and Intelligent Interaction (Vol. 6975, pp. 578-587): Springer Berlin Heidelberg.

Pew Research Centre. (2014). Device Ownership Over Time Retrieved August 28, 2014, from http://www.pewinternet.org/data-trend/mobile/device-ownership/ Preziosa, A., Grassi, A., Gaggioli, A., \& Riva, G. (2009). Therapeutic applications of the mobile phone. British Journal of Guidance \& Counselling, 37(3), 313-325. doi: 10.1080/03069880902957031

Proudfoot, J., Clarke, J., Birch, M.-R., Whitton, A. E., Parker, G., Manicavasagar, V., ... Hadzi-Pavlovic, D. (2013). Impact of a mobile phone and web program on symptom and functional outcomes for people with mild-to-moderate depression, anxiety and stress: a randomised controlled trial. BMC psychiatry, 13(1), 312.

Rahman, M. M., Ali, A. A., Plarre, K., al'Absi, M., Ertin, E., \& Kumar, S. (2011). mConverse: inferring conversation episodes from respiratory measurements collected in the field. Paper presented at the Proceedings of the 2nd Conference on Wireless Health, San Diego, California. 
THE SMART THERAPIST AND mHEALTH TECHNOLOGIES

Reger, G. M., Hoffman, J., Riggs, D., Rothbaum, B. O., Ruzek, J., Holloway, K. M., \& Kuhn, E. (2013). The “PE coach” smartphone application: An innovative approach to improving implementation, fidelity, and homework adherence during prolonged exposure. Psychological Services, 10(3), 342-349. doi: 10.1037/a0032774

Riley, W. T., Glasgow, R. E., Etheredge, L., \& Abernethy, A. P. (2013). Rapid, responsive, relevant (R3) research: a call for a rapid learning health research enterprise. Clinical and translational medicine, 2(1), 1-6.

Shuger, S. L., Barry, V. W., Sui, X., McClain, A., Hand, G. A., Wilcox, S., . . B Blair, S. N. (2011). Electronic feedback in a diet-and physical activity-based lifestyle intervention for weight loss: a randomized controlled trial. Int J Behav Nutr Phys Act, 8(41), 1-8.

Shyamkumar, P., Oh, S., Banerjee, N., \& Varadan, V. K. (2013). A wearable remote brain machine interface using Smartphones and the mobile network. Advances in Science and Technology, 85, 11-16. doi:

\subsection{8/www.scientific.net/AST.85.11}

Sims, H., Sanghara, H., Hayes, D., Wandiembe, S., Finch, M., Jakobsen, H., . . . Kravariti, E. (2012). Text message reminders of appointments: a pilot intervention at four community mental health clinics in London. [Evaluation Studies;]. Psychiatric services (Washington, D.C.), 63(2), 161-168.

Swan, M. (2012). Sensor mania! the internet of things, wearable computing, objective metrics, and the quantified self 2.0. Journal of Sensor and Actuator Networks, 1(3), 217-253. 
THE SMART THERAPIST AND mHEALTH TECHNOLOGIES

Tilley, P. J. M., \& Rees, C. S. (2014). A clinical case study of the use of ecological momentary assessment in obsessive compulsive disorder. Frontiers in Psychology, 5, 1-8. doi: 10.3389/fpsyg.2014.00339

Turner, W. A., \& Casey, L. M. (in press). Outcomes associated with virtual reality in psychological interventions: Where are we now? Clinical Psychology Review.

Watts, S., Mackenzie, A., Thomas, C., Griskaitis, A., Mewton, L., Williams, A., \& Andrews, G. (2013). CBT for depression: a pilot RCT comparing mobile phone vs. computer. BMC psychiatry, 13(1), 49. 